19 Revue d'histoire du XIXe siècle

Société d'histoire de la révolution de 1848 et des

révolutions du XIXe siècle

$20 / 21 \mid 2000$

Varia

\title{
La notion d'insalubrité. Un processus de rationalisation 1850-1902
}

Yankel Fijalkow

\section{OpenEdition}

\section{Journals}

Édition électronique

URL : https://journals.openedition.org/rh19/213

DOI : $10.4000 /$ rh 19.213

ISSN : $1777-5329$

Éditeur

La Société de 1848

\section{Édition imprimée}

Date de publication : 1 juin 2000

Pagination : 135-156

ISSN : 1265-1354

Référence électronique

Yankel Fijalkow, «La notion d'insalubrité. Un processus de rationalisation 1850-1902 », Revue d'histoire du XIXe siècle [En ligne], 20/21 | 2000, mis en ligne le 06 décembre 2016, consulté le 21 septembre 2021. URL : http://journals.openedition.org/rh19/213 ; DOI : https://doi.org/10.4000/rh19.213

Ce document a été généré automatiquement le 21 septembre 2021.

Tous droits réservés 


\title{
La notion d'insalubrité. Un processus de rationalisation 1850-1902
}

\author{
Yankel Fijalkow
}

1 L'apparition de la loi de 1850 dans le dispositif juridique français s'intégre dans un mouvement européen d'inquiétude face aux conditions d'habitat des populations laborieuses. Mais les ambitions du texte français semblent bien modestes comparées à celles prévalant en Angleterre où, en 1834, la loi sur .les pauvres permet le vote de textes importants relatifs à l'Amélioration de la Ville (1847), à la Santé Publique (1848), et au surpeuplement (loi sur les garnis de 1851). Ces textes se préoccupaient de l'alimentation en eau courante, de la ventilation et des égouts dans les constructions neuves, permettant même aux collectivités locales de se substituer aux propriétaires privés pour les bâtiments existants. En France, la loi de 1850 sur les logements insalubres, volontairement imprécise dans la définition de l'insalubrité, est libérale pour ce qui concerne l'interprétation de cette notion et les pouvoirs nouveaux accordés aux municipalités. L'ambition nationale du texte, conjuguée à la mobilisation des notables et des pouvoirs locaux fait donc de la loi de 1850, un texte radicalement nouveau en raison des principes et des mécanismes auquel il conduit. Paradoxalement, les villes qui se trouvent amenées à définir localement l'insalubrité contribuent à la rationalisation, technicisation, normalisation de la notion. Un processus d'adaptation, d'ajustement local débute dès la publication de la loi à partir du moment où celle-ci est interprétée et intégrée à l'action municipale ${ }^{1}$. Ce processus explique en partie pourquoi, en définitive, la loi s'est peu appliquée dans les villes et est restée au stade expérimental ${ }^{2}$. On aurait tort, néanmoins, d'en minimiser les effets : la loi de 1850 concrétise pour la première fois en France l'apparition d'un nouvel objet de préoccupation sociale : le logement, le ménage, l'habitat. Ainsi, nous voulons montrer dans cet article comment le terme d'insalubrité, inventé dans des circonstances précises par le législateur, fait l'objet dans les cinquante années suivantes d'un travail de définition, mené par l'ensemble de la société et des corps professionnels qui puisent une légitimité dans cette nouvelle catégorie ${ }^{3}$. 


\section{La diversité des définitions de l'insalubrité}

\section{En rappel de la loi}

2 La loi de 1850 n'est pas le premier texte concernant l'insalubrité. La loi de 1807 relatif au dessèchement des marais, aux travaux des rues, places et quais dans les villes et de salubrité dans les communes s'était donné très tôt pour objectif d'assainir les localités, c'est-à-dire d'imposer aux municipalités la réalisation de plans d'alignement et d'extension destinés à limiter les effets nocifs de certains milieux naturels et le laisserfaire des propriétaires privés. Dans ce cadre, les compétences professionnelles des ingénieurs des Ponts et chaussées, des Mines ou de l'École centrale des arts et manufacture, créé en 1829, avaient été mobilisées par les élites locales. De même un arrêté du 18 décembre 1848 avait créé, à la suite de l'exemple parisien initié dès 1802, des conseils d'hygiène et de salubrité dans les départements au niveau du canton et de l'arrondissement. Composés de huit à quinze membres bénévoles nommés par le préfet, ils devaient être consultés sur des problèmes aussi divers que l'assainissement des localités et des habitations, les maladies épidémiques et endémiques, la qualité des aliments et des eaux, les grands travaux d'utilité publique ${ }^{4}$. Ainsi, si l'on excepte le décret du 15 décembre 1810 relatif aux ateliers qui répandent une odeur insalubre ou incommode, la loi de 1850 représente le premier texte qui s'attache directement à la notion d'insalubrité indépendamment de son antonyme, la salubrité.

\section{Que signifie alors cette notion d'insalubrité}

Selon la loi de 1850, « les logements réputés insalubres se trouvent dans des conditions de nature à porter atteinte à la vie ou à la santé de leurs habitants ». En soulignant que l'insalubrité relèverait de la nature du logement (mode de construction, humidité, ventilation, etc.) la loi de 1850 s'écarte du rôle fondamental des municipalités établies depuis la Révolution en matière de sécurité et de salubrité : «Faire jouir les habitants d'une bonne police notamment de la propreté, de la sûreté et de la tranquillité dans les rues, lieux et édifices publics». En effet, contrairement aux usages, le pouvoir local s'attachera moins à la surveillance du milieu environnant qu'à celle de la cellule habitée.

Pourtant l'application de la loi dans les communes et les départements a fortement varié selon les rapports de force locaux. D'une part en raison du caractère facultatif de la loi, les conseils municipaux pouvant nommer une commission des logements insalubres. D'autre part en raison de la composition de ces commissions présidées par le maire: cinq à neuf membres bénévoles, dont au moins un médecin, un architecte ou tout autre homme de l'art, un membre du bureau de bienfaisance et du conseil de prud'hommes. Cette liste des compétences à réunir sur le plan médical, social et constructif, laisse aux conseils municipaux une liberté de choix d'autant plus grande qu'ils peuvent élargir jusqu'à neuf le nombre des membres, permettant éventuellement de s'ouvrir aux avis des grands propriétaires de la commune. La qualité et le nombre des membres de la commission s'avèrent d'autant plus importante que celle-ci se trouve placée par la loi dans une position d'arbitre entre le locataire plaignant et le propriétaire ${ }^{5}$. En effet, l'action de la municipalité n'est possible qu'à la suite de la visite sur place de la commission ${ }^{6}$, celle-ci pouvant alors proposer au conseil municipal d'obliger les propriétaires à réaliser des 
.travaux d'assainissement ou d'interdire la location à titre d'habitation (article 5). Ceci est d'autant plus vrai que les propriétaires bénéficient d'une procédure doublement contradictoire: dès le premier rapport de la commission des logements insalubres le propriétaire dispose d'un mois pour noter ses observations à la mairie; de même peut-il à l'issue de la décision du conseil municipal déposer un recours, suspensif, auprès du conseil de préfecture. Après ce recours auprès du conseil de préfecture le propriétaire pourra même intercéder en appel en conseil d'État, si le logement, reconnu non susceptible d'assainissement, est alors interdit à l'habitation par l'autorité municipale. Mais comment ce cas de figure pourrait-il se multiplier sachant que le locataire plaignant pourrait alors voir son bail résilié sans aucun dommage-intérêt? En définitive, l'insalubrité, dénoncée par la plainte d'un locataire, évaluée par des experts locaux, et dénoncée par le pouvoir politique se trouve limitée par les nombreuses possibilités de recours juridiques des propriétaires.

Une telle procédure, forcément longue et sans issue certaine, n'obéit pas au même souci d'utilité publique que l'autre type d'insalubrité suggérée par le texte: l'insalubrité extérieure et permanente. La loi distingue en effet l'insalubrité provenant de causes inhérentes à l'habitation, soumise au régime précédemment décrit, et l'insalubrité extérieure et permanente, plutôt soumise à l'expropriation. Alors que les causes inhérentes à l'habitation relèvent des défauts du logement (pièces sans jour, approvisionnement en eau, systèmes d'évacuation, surpeuplement), l'insalubrité extérieure et permanente représente tout ce qui fait de l'espace urbain un milieu nocif et requiert une intervention de la commune par l'expropriation. En outre, les propriétés insalubres comprises dans un périmètre de travaux peuvent également faire l'objet d'acquisitions forcées : « Lorsque l'insalubrité est extérieure et permanente ou lorsque ces causes ne peuvent être détruites que par des travaux d'ensemble, la commune pourra acquérir la totalité des propriétés comprise dans le périmètre des travaux » (article 13).

\section{L'application de la loi dans les départements}

6 On retiendra ici que la philosophie de la loi de 1850 est fondamentalement localiste. Les commissions de logements insalubres sont formées des «experts » de la commune qui examinent logement par logement et selon les règles locales, supposées relever d'une connaissance intime du milieu, l'insalubrité dont se plaignent auprès d'elles les locataires. Dès lors, le rapport réalisé en 1858 par le ministre de l'Agriculture, du Commerce et des Travaux Publics ${ }^{7}$, à la suite d'une enquête auprès des préfets, témoigne d'une profonde inégalité d'application de la loi: 11 départements l'ont fidèlement appliquée, 26 départements ont tenté de la mettre en œuvre, 30 n'ont pas compris son objet, 19 départements n'ont pas même répondu à l'enquête du ministère. 


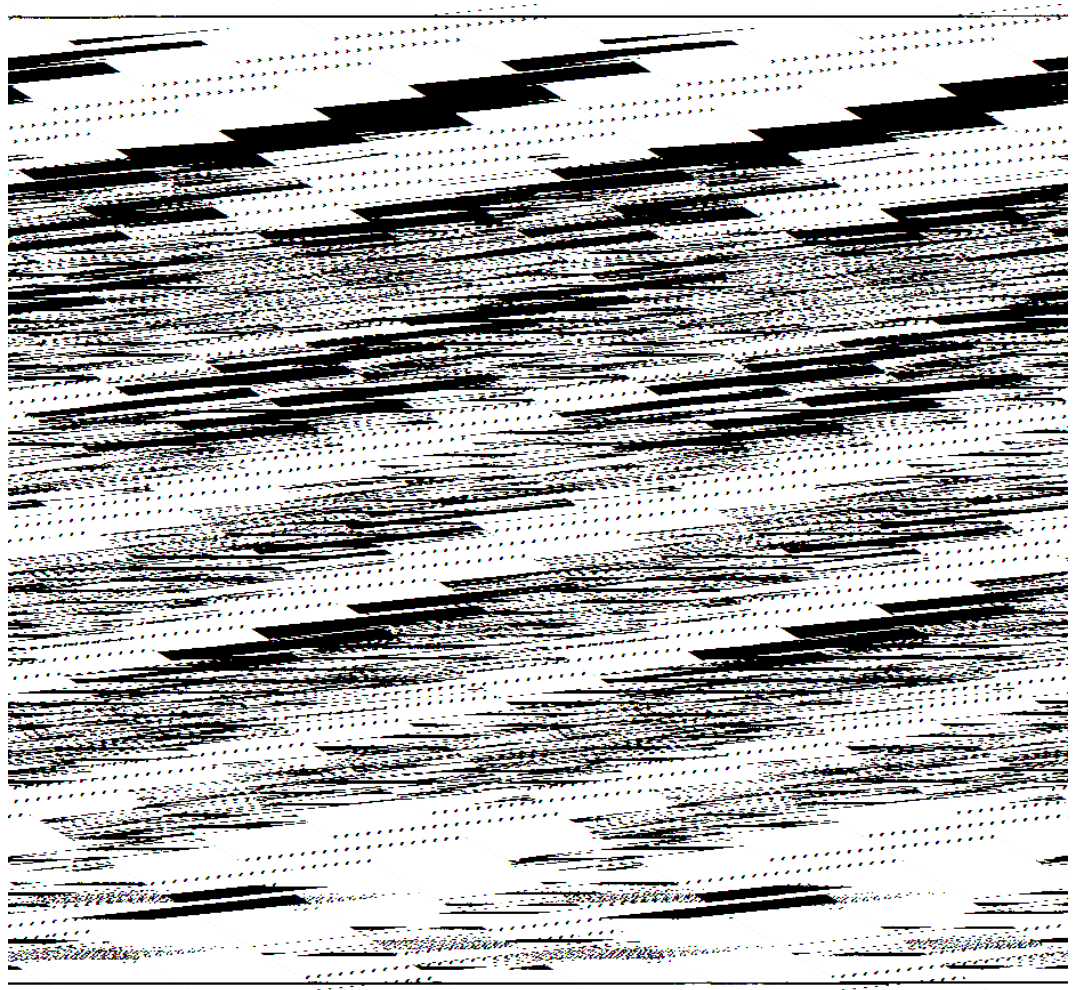

(c) Yankel Fijalkow, 2000

7 L'enquête montre en effet que la loi s'est surtout traduite par des actes dans les départements du nord et de l'ouest, dans le Rhône, la Gironde, la Moselle, Dans le nord où près de 400 communes ont désigné une commission des logements insalubres, l'inquiétude s'attache d'abord aux logements souterrains et à l'humidité. À Lille, en 1858, les Commissions ont déjà visité plus de 4000 logements : elles préconisent volontiers de remplacer le sol à peine battu des habitations par du carrelage, d'ouvrir de nouvelles fenêtres, de blanchir les murs à la chaux, de mieux nettoyer les latrines. Mais peu d'affaires semblent conduire les propriétaires devant des tribunaux. Même si des injonctions de réaliser certains travaux leur sont signifiées, il semble tout de même plus pertinent de faire endosser la responsabilité de l'insalubrité au locataire. Ainsi, le rapport d'enquête ministériel, se félicite-t-il des initiatives du département du Nord qui «a compris que quand il s'agit du bien-être et de la moralisation du pauvre, mieux vaut souvent encourager que réprimer et dans un nombre de communes assez notable une prime fournie souvent par le bureau de bienfaisance est distribuée annuellement aux ouvriers qui entretiennent leur logement avec soin et propreté ». Cette manière habile de tourner la loi, sous couvert de moralisation et de générosité n'est pas relevée par le ministre ${ }^{8}$. Aussi n'est-on guère surpris de relever qu'à Lyon, où l'on regrette que la commission n'ait visité qu'un nombre limité de logements en raison de l'inviolabilité du domicile, le ministre tienne à rappeler que: «la loi de 1850, faite pour protéger le locataire contre un propriétaire indifférent, permet aussi de défendre le locataire luimême contre sa propre incurie ». Si la loi de 1850 n'a pas considéré le locataire comme un acteur principal, l'éventualité que des propriétaires puissent porter plainte, sous couvert de moralité, contre un « locataire insalubre » n'a pas été totalement exclue. Il est possible, 
dans ce cas de figure, de porter plainte auprès d'une commission d'hygiène et de salubrité.

Sur le plan technique, le ministre est fortement présent par ses recommandations. Il demande au maire de Boulogne, qui impose aux propriétaires la construction de fosses d'aisance, si «c'est bien le meilleur système à adopter " alors que la ville de Douvres le rejette avec vigueur. Les efforts des villes comme Rennes et Saint-Malo, où l'on prescrit des conduits d'évacuation des eaux, sont couronnés. À Nantes, où près de mille maisons ont été visitées, les moyens indiqués qui consistent en cloisons d'isolement et revêtements hydrofuges semblent pertinents. À Bordeaux la destruction des hôtels des miracles «tristes demeures où le pauvre trouve un gîte pour dix ou vingt centimes par nuit, se composent souvent de cellules petites et mal aérées quelquefois en contrebas du sol ou privées de jour» est encouragée. L'État paraît alors sous un jour très interventionniste. Néanmoins il fustige avec vigueur toute proposition qui conduirait à augmenter ses charges, ainsi que le propose le préfet de la Seine-Inférieure, lorsqu'il énumère les dépenses que l'État devrait supporter pour faire disparaître les causes d'insalubrité de la ville d'Eu.

$\mathrm{Au}$ total, nous n'avons guère l'impression d'une unité dans les injonctions de l'État sur le plan technique bien que les causes d'insalubrité relevant de l'humidité soient le plus souvent citées. L'idée que des diagnostics différents de l'insalubrité s'attachent à des traitements spécifiques selon les localités est affirmée par la loi. Mais on regrette une "grande uniformité des moyens d'assainissement à peine modifiée par les ressources inhérentes à chaque localité » alors qu'ils devraient varier selon les habitudes locales. Ainsi, lorsque le département du Nord propose la création d'une institution supérieure chargée de coordonner les avis des commissions locales, le ministre rappelle que le caractère local de loi relève d'une nécessaire "discrétion", certainement eu égard aux propriétaires. Néanmoins, l'objectif poursuivi est avant tout d'encourager les communes à nommer des commissions de logements insalubres quels que soient les remèdes qu'elles proposent. Cette absence de finalité explique, au moins en partie, la réticence des municipalités à s'engager « malgré des taux élevés de mortalité »: « peu d'insalubrité et pas d'argent ».

\section{L'évolution de la loi, des échelles et des objets de l'insalubrité à Paris}

10 La commission des logements insalubres de Paris est souvent citée comme modèle. Le rapport de 1858, montre même comment la diffusion de ses rapports dans les autres départements a convaincu le préfet du Gard du bien-fondé de la loi. Aussi, l'évolution de la notion d'insalubrité et l'émergence de nouveaux objets insalubres à Paris semble révélatrice de l'évolution des esprits.

Dans ses premiers jours d'exercice, la commission des logements insalubres de Paris est profondément "aériste ", attentive aux miasmes et aux odeurs. Elle n'admet sa compétence que dans la sphère intime de l'appartement : « Il y a insalubrité partout où il y a mauvaises odeurs pouvant vicier l'air de l'habitation. Cette insalubrité peut être extérieure : amas d'immondices, stagnation d'eau pour cause de mauvais pavage, défaut d'entretien des conduites d'eau ménagères, mauvaise odeur des fosses et cabinets, saletés des murs, corridors et escaliers. Cette insalubrité extérieure n'entre pas dans la compétence de la loi de $1850 »^{10}$. Ainsi, l'insalubrité intérieure, inhérente à l'habitation, se 
manifeste par «l'agglomération des habitants, l'humidité, le défaut d'air et de lumière, l'exiguïté des logements, la malpropreté $»^{11}$.

Dans le cadre restreint de l'univers domestique, la commission impose ainsi aux propriétaires le nettoiement, l'ouverture d'une fenêtre, la création d'un vasistas, la suppression de cloisons, le pavage. Mais elle souligne rapidement son impuissance devant le surpeuplement : aérer le logement sans tenir compte de la taille des appartements et du nombre d'habitants par foyer, relève parfois de l'absurde. L'absence d'eau dans les maisons comme cause d'insalubrité est évoquée, sans doute sous l'influence de l'ordonnance de Police du 23 novembre 1853 relative à la salubrité des habitations ${ }^{12}$. Enfin, la commission se déclare impuissante face à certaines agglomérations urbaines. Dans les rues étroites et sinueuses du $12^{\mathrm{e}}$ arrondissement de l'époque (rues Saint-Jacques, Jardin des Plantes, Saint-Michel) « rien ne changera si l'on n'entreprend pas de grands travaux $»^{13}$.

En 1859, le pouvoir des commissions de logements insalubres est renforcé. Avant cette date, la plainte d'un locataire pouvait autant s'acheminer vers la préfecture de police et ses commissions de quartiers formées depuis 1832 sous l'autorité de la vieille commission d'hygiène et de salubrité (créée en 1802) que vers la récente commission des logements insalubres issue de la loi de 1850. À partir du décret du 24 octobre 1859, les pouvoirs du préfet de police sont strictement limités aux hôtels meublés et garnis. En dehors de ceuxci, la commission d'hygiène ne peut in fine que renvoyer les affaires à la commission des logements insalubres. C'est dans cette période que l'on voit progresser l'intérêt pour la notion d'insalubrité. En effet, dans le rapport de 1857, la commission des logements insalubres déplore « les habitudes invétérées et l'insouciance de gens qui ne comprennent pas la portée des conseils et pour lesquels le mot d'INSALUBRITÉ est vide de sens $»^{14}$. À cette époque, le nombre des affaires traitées s'élève à environ 500 , chiffre bien inférieur à celui de 4000 (un record) qui permet à la commission de 1866 de considérer que son action est désormais entrée dans les mœurs. Après cette période d'intense activité le rythme annuel se stabilise autour de 2 500-3 000 affaires.

Nombre d'affaires présentées à la Commission des logements insalubres

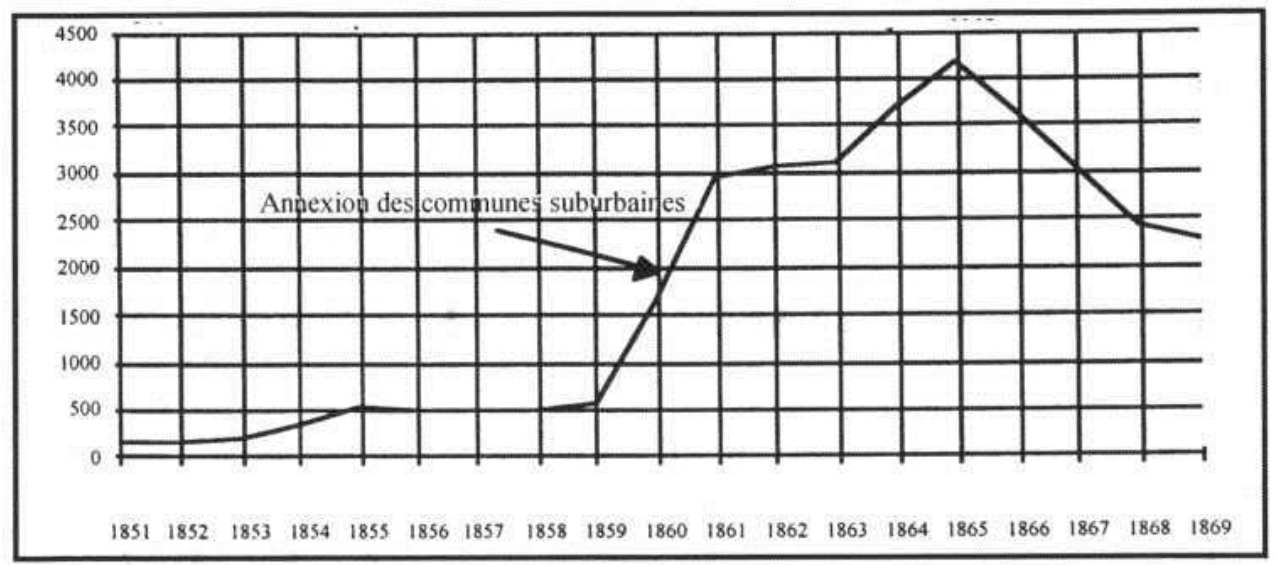

L'annexion des communes suburbaines de Paris en 1859 est l'une des explications possibles du gonflement du volume des affaires traitées. Ce changement de volume implique une modification de la commission des logements insalubres qui est décidée par arrêté du préfet de la Seine le 2 mars 1860. Désormais, la commission se compose de sept membres ès qualités (parmi lesquels des ingénieurs des Ponts et chaussées, des directeurs 
de services municipaux des eaux et de la voirie), 20 membres désignés par le conseil municipal dans chaque arrondissement, cinq personnalités qualifiées également désignées par le conseil municipal (un architecte, un professeur de l'école de Turgot, un ancien juge au tribunal de commerce, un médecin vérificateur des décès).

Ce changement organisationnel est le reflet des nouvelles préoccupations de la commission. Les loges de portier, les baraquements de banlieue, les maisons nouvellement construites, constituent une part importante des interventions. On s'évertue par exemple à rechercher dans les baraquements de la petite banlieue qui, du locataire du terrain ou du propriétaire, doit supporter les travaux de réduction de l'insalubrité. Cherchant à s'échapper des cas isolés, la commission rapporte volontiers ces faits à d'autres espèces proches, en terme de quartiers, de rues, de cités ou d'immeubles : rues des Filles-Dieu $\left(11^{\mathrm{e}}\right)$ Sainte-Marguerite $\left(12^{\mathrm{e}}\right)$, Jacques-Cartier $\left(13^{\mathrm{e}}\right)$, les cités Jeanned'Arc $\left(13^{\mathrm{e}}\right)$, Bablot $\left(13^{\mathrm{e}}\right)$, des Kroumirs (19e). La cité Jeanne d'Arc (dix immeubles, 2000 habitants et 1200 logements sans éviers) est un cas connu de la commission, les propriétaires multipliant les appels jusqu'en Conseil d'État: leur résistance aux injonctions de la commission des logements insalubres durera jusqu'en 1930. Plus problématique est le cas des cités comme celles des Kroumirs, Gand, Philippe, du Tarn (19 e) qui consistent généralement en un agglomérat de cabanes à un étage en planches et plâtras, exploité par un principal locataire. Est-ce lui que la commission devrait sanctionner? Il reste enfin, si l'on excepte les garnis qui font l'objet de nombreux rapports mais relèvent du conseil d'hygiène et de la compétence du préfet de Police, les cités de chiffonniers qui consistent également en cabanes de fortune louées à la semaine (Clos Macquart, $19^{\mathrm{e}}$, Sainte Marguerite, $11^{\mathrm{e}}$ ). Ces agglomérations posent des problèmes urbains qui sont aussi traités par le texte de 1852 relatif aux rues de Paris que nous étudierons plus loin.

\section{Nouvelles professions face à l'insalubrité}

16 Après l'épisode de la Commune, le ton accommodant de la commission des logements insalubres change peu à peu sous l'effet d'un conseil municipal élu depuis 1871 et de rapporteurs plus véhéments, annonçant les premières escarmouches de la «bataille du tout-à-l'égout ». La commission des logements insalubres n'est plus présidée par un médecin ${ }^{15}$. Persuadée de l'expansion prochaine de ses pouvoirs, elle durcit sa doctrine, élabore des projets de règlements de construction, propose des mesures qui lui valent l'hostilité des syndicats de propriétaires. La mauvaise construction originaire, les modifications opérées sans contrôle à l'intérieur des bâtiments, le défaut d'entretien sont relevés avec force. Il s'agit désormais de préserver le bâti contre l'humidité, de permettre un renouvellement de l'air grâce à un cubage adéquat, de se préserver des émanations provenant des fosses, de permettre l'écoulement des eaux pluviales et ménagères. Dès lors, la définition de l'insalubrité s'élargit, recouvre le champ de l'habitabilité et parfois même du confort, si bien que le devoir social du propriétaire se trouve investi de nouvelles obligations ${ }^{16}$. Le souci d'une intervention publique forte est confirmé. Il s'exerce sur les équipements sanitaires (eau salubre et fosses fixes), les réseaux et la solidité des constructions: «Les causes de l'insalubrité les plus fréquentes et les plus graves sont l'humidité, le défaut d'air et de jour, l'absence d'eau salubre, le mauvais état des planchers, des parquets ou des carrelages, les infiltrations par les toitures, l'exiguïté des pièces habitées, l'encombrement, l'insuffisance de hauteur des plafonds, l'absence de 
cheminées, le défaut de tirage des tuyaux de fumée, la mauvaise installation des fosses fixes $»^{17}$. insalubres vont trouver une solution juridique en appliquant le décret de 1852 relatif aux rues de Paris. Ce décret de 1852 reprend à son compte l'exigence de salubrité : « Dans tout projet d'expropriation pour l'élargissement, le redressement ou la formation des rues, l'administration aura la faculté de comprendre la totalité des immeubles atteints et lorsqu'elle jugera que les parties restantes ne sont pas d'une étendue ou d'une forme qui permette d'y élever des constructions salubres. Elle pourra comprendre dans l'expropriation des immeubles en dehors de l'alignement lorsque leur acquisition sera jugée nécessaire pour la suppression d'anciennes voies publiques jugées inutiles. Les parcelles de terrain acquises en dehors des alignements et non susceptibles de recevoir des constructions salubres seront réunies aux propriétés contiguës soit à l'amiable soit par l'expropriation de ces propriétés » (article 2).

dexte est moins de détruire des constructions dont l'insalubrité est extérieure et permanente que d'édifier lorsque la configuration des lieux s'y prête, des immeubles salubres. À l'articulation des deux textes, 1850 et 1852, émergent de nouvelles compétences pour les agents-voyers dont le premier cadre réglementaire provient d'un édit de 1607. Les textes de 1850 et 1852 les chargent désormais des procédures d'alignement et d'élargissement des voies, du nivellement (article 4), de la prédisposition des constructions nouvelles à accueillir l'égout (article 6), et des règles d'élévation. Disposés désormais à accueillir les autorisations de construire sous l'angle de la «sûreté publique et de la salubrité ", les agents-voyers sont peu à peu associés aux commissions de logements insalubres et aux conseils départementaux d'hygiène et de salubrité. Alors que les textes juridiques sur l'insalubrité s'attachent à la notion de milieu (loi de 1807) et de logement (loi de 1850), les agents-voyers attirent l'attention sur l'immeuble, interstice 
entre le domaine public et privé, mais aussi lieu d'arrivée des réseaux sanitaires comme le constatent les ingénieurs.

21 Ainsi, les années 1880 offrent une grande diversité de regards techniques de plus en plus professionnels sur l'insalubritée ${ }^{1}$. Alors qu'on reconnaît à l'initiative haussmannienne un dispositif mal adapté à ses visées, un médecin comme André-Justin Martin et un ingénieur comme Alfred Durand-Claye s'associent pour entreprendre une véritable action contre l'insalubrité des immeubles et l'aménagement des quartiers bien connus pour leur surmortalité. Le discours d'Alphand donné à l'académie des Beaux-Arts en 1891 illustre bien la rupture politique qui accompagne cette plus grande attention à l'habitat et aux réseaux d'assainissement: " Les travaux apparents tels que ceux que je viens de passer brièvement en revue et qui répondent aux besoins extérieurs de l'existence ne suffisent pas à la vie d'une grande cité comme Paris; il lui faut encore un organisme intérieur, comme dans le corps humain et de la perfection de cet organisme dépend la santé de la population. Le réseau d'égouts qui n'était que de 228 kilomètres en 1860 avait atteint 18 ans plus tard 600 kilomètres et avait pris l'unité nécessaire à un corps dont toutes les parties, collecteurs et petites galeries, étaient agencées de manière à concourir au même objet $»^{22}$.

22 A Paris, les auteurs de cette révolution des modes de pensée sont bien les commissions des logements insalubres. Désignées par le conseil municipal, lui-même élu depuis 1871, elles sont devenues plus libres de leurs propositions. Leur optique, centrée sur le logement et la famille, est couramment adoptée. Elle peut s'opposer à une perspective plus technique. En effet, on pense de plus en plus qu'une opération importante sur le cadre et sur l'environnement pourra venir à bout des épidémies successives. L'air et la lumière pénétreront dans les bâtiments et chasseront les miasmes qui les hantent. $\mathrm{Si}$ l'action ponctuelle menée grâce à la loi de 1850 est reconnue inefficace, on imagine déjà le soutien d'une initiative charitable pour résoudre le problème de l'insalubrité. Mais celleci est encore très marginale. Amener le tout-à-l'égout et l'eau, ne serait-ce que dans les cours, constitue déjà un objectif important, comme éviter les constructions hâtives ou respecter une échelle entre la dimension des voies et la hauteur des bâtiments. Il faut donc réformer la loi de 1850, donner aux pouvoirs publics une emprise globale sur les propriétés, cesser de considérer l'insalubrité à l'échelle d'appartements isolés. Penser globalement, faire de la statistique et... de l'urbanisme !

\section{L'efficacité discrète de la loi de 1850 et son évolution}

Si l'insalubrité a changé de définition et s'est assortie de nombreuses compétences, le parc immobilier, à Paris comme dans d'autres villes, n'a guère à se féliciter des résultats obtenus sur le plan de la salubrité du parc. Pourtant, si les effets juridiques de la loi de 1850 sont finalement bien modestes, sa portée est peut être ailleurs, dans l'évolution des esprits, qui conduit à deux visions de l'insalubrité centrée sur le ménage ou sur le milieu.

\section{Rationaliser l'étude du logement en harmonisant les jugements sur l'insalubrité}

À Paris, quelques années après l'annexion des communes suburbaines, les questions relatives à l'homogénéité des jugements relatifs au diagnostic d'insalubrité sont toujours entières. En 1879, le statisticien Toussaint Loua ${ }^{23}$ montre que depuis 1850 la commission 
des logements insalubres de Paris a examiné 50717 logements. Si le nombre total de maisons s'établit à 71920 dans la capitale, déduction faite des propriétaires occupant seuls leur immeuble, chaque maison a été visitée au moins une fois. Par ailleurs 100000 habitants résident dans des logements insalubres si l'on considère que l'Assistance Publique est, avec le concours des médecins de bienfaisance, venue en aide à 39.604 ménages dont $15 \%$ ont des loyers inférieurs à 100 francs et $52 \%$ entre 100 et 200 francs. Ces 39604 logements abritent 101749 indigents. Si l'on admet le rapprochement audacieux qui conduit à assimiler la population indigente et les logements insalubres, les taudis parisiens représentent $6 \%$ du parc et un habitant sur 20 .

Ces chiffres sont importants car ils traduisent le rythme d'activité de la commission des logements insalubres. Néanmoins ce travail est-il efficace? En 1883, sur 2498 plaintes reçues émanant de locataires, 1428 soit $56 \%$ ont donné lieu à visites et rapports au conseil municipal, c'est à dire ont été considérées comme relevant vraiment de l'insalubrité. 855 , soit $34 \%$ n'ont donné lieu qu'à des visites seules ${ }^{24}$. On peut donc dire qu'au-delà des possibilités de recours dont disposent les propriétaires, l'un des enjeux de la loi réside dans l'interprétation de l'insalubrité qui confronte l'avis des locataires à celle de la commission. En 1883, $75 \%$ des plaintes de locataires habitant le $16^{\mathrm{e}}$ arrondissement sont déclarées recevables contre $40 \%$ dans les $19^{\mathrm{e}}$ et $20^{\mathrm{e}}$, mais seulement $25 \%$ dans le $8^{\mathrm{e}}$. Ces fortes variations géographiques seraient à étudier en détail pour illustrer éventuellement comment la nature des rapports sociaux à l'œuvre entre propriétaires et locataires a pu influencer les commissions, même s'il semble difficile d'expliquer systématiquement leurs jugements par le «niveau social » des arrondissements ${ }^{25}$.

Plaintes déposées auprès de la commission des logements insalubres ${ }^{26}$

\begin{tabular}{|c|c|c|c|}
\hline Arrondis. & $\begin{array}{c}\text { Plaintes } \\
\text { reçues }\end{array}$ & $\begin{array}{c}\text { Plaintes soumises au conseil } \\
\text { municipal }\end{array}$ & $\begin{array}{c}\text { Pourcentage de plaintes } \\
\text { recevables }\end{array}$ \\
\hline 1 & 85 & 38 & $45 \%$ \\
\hline 2 & 175 & 130 & $53 \%$ \\
\hline 3 & 66 & 35 & $36 \%$ \\
\hline 4 & 145 & 52 & $71 \%$ \\
\hline 5 & 87 & 62 & $60 \%$ \\
\hline 6 & 93 & 54 & $25 \%$ \\
\hline 7 & 99 & 59 & $37 \%$ \\
\hline 8 & 48 & 12 & $79 \%$ \\
\hline 10 & 136 & 53 & $64 \%$ \\
\hline 11 & 143 & 107 & 52 \\
\hline
\end{tabular}




\begin{tabular}{|c|c|c|c|}
\hline 12 & 147 & 95 & $65 \%$ \\
\hline 13 & 71 & 33 & $46 \%$ \\
\hline 14 & 98 & 71 & $72 \%$ \\
\hline 15 & 177 & 114 & $64 \%$ \\
\hline 16 & 73 & 55 & $52 \%$ \\
\hline 17 & 145 & 75 & $60 \%$ \\
\hline 18 & 285 & 172 & $43 \%$ \\
\hline 19 & 136 & 58 & $42 \%$ \\
\hline 20 & 145 & 61 & $56 \%$ \\
\hline Total & 2498 & 1428 & \\
\hline
\end{tabular}

Quoi qu'il en soit ces écarts de jugements ne laissent pas d'inquiéter certains médecins tels Octave Du Mesnil qui finissent par remettre en cause l'un des principes de la loi de 1850. En effet, ce fidèle successeur de Villermé à la rédaction des Annales d'hygiène publique et de médecine légale recherche en 1878 une définition universelle du mauvais logement nécessaire pour rationaliser l'action publique. Cette idée, exprimée dans un article paru dans les Annales d'hygiène publique et de médecine légale ${ }^{27}$ se traduit par la tentative de systématisation des rapports soumis à la commission des logements insalubres sous la forme de 17 questions établissant une description précise des lieux : nombre d'habitants dans l'immeuble et par chambre, description de l'immeuble insistant particulièrement sur l'aération, la ventilation, l'état des sols, la description des chambres en termes d'éclairage, le mode d'écoulement des matières usées, la présence d'industrie... Ce questionnaire rompt radicalement avec l'idée que l'insalubrité ne peut faire l'objet de normes universelles et se rapproche de l'idée de moyenne développée par Quételet et Villermé.

Cette posture critique à l'égard de la loi de 1850, Du Mesnil l'introduit à nouveau le 25 octobre 1881 à la Société de médecine publique dans une brochure intitulée L'habitation du pauvre à Paris ${ }^{28}$. Il mène alors un débat sans concession sur le percement des rues initié par Haussmann: "Quand il s'est agi du percement du boulevard Saint-Michel ou de l'ouverture de l'avenue de l'Opéra et de la disparition de la Butte aux Moulins, on ne parait pas s'être préoccupé de ce que deviendrait toute cette catégorie d'habitants pauvres qui vivaient dans des immeubles sordides du vieux Paris, alors que par suite de l'expropriation des immeubles sacrifiés ils seraient mis en demeure de chercher un abri. De là deux faits également graves au point de vue de la salubrité des habitations parisiennes. Le premier c'est que l'encombrement dans les logements d'ouvriers a augmenté [...]. L'autre conséquence également funeste de cette imprévoyance a été l'édification sur des terrains vagues à la limite de l'ancienne enceinte d'une quantité énorme de constructions improvisées [...]. On n'a donc pas diminué en réalité l'insalubrité des maisons de Paris. On s'est borné à déplacer le foyer et à l'écarter plus ou moins des centres primitifs assurant des travaux pour un demi-siècle aux commissions des 
logements insalubres de l'avenir ${ }^{29}$. Et Du Mesnil expose en guise de démonstration la baisse entre 1877 et 1880 de la population indigente au centre de Paris (neuf premiers arrondissements) comparée à une forte augmentation (5\% par an) dans les arrondissements de l'est. Cette analyse, certainement trop systématique, donne la tonalité des réflexions à venir : rechercher une plus grande uniformité dans les jugements portant sur l'insalubrité, se préoccuper des effets sociaux notamment du surpeuplement des ménages ouvriers.

\section{La question du surpeuplement}

Ces réflexions de la période post haussmanienne en faveur d'une rationalisation des jugements d'insalubrité se traduisent par la mise en œuvre du recensement de 1891 à Paris qui prend en compte pour la première fois en France la question du surpeuplement. Celle-ci est décidée par la commission permanente de statistique municipale, dont le secrétaire est Jacques Bertillon, docteur en médecine, et fils du démographe LouisAlphonse Bertillon (1821-1883). C'est dans une séance du 25 octobre 1881, qui réunit entre autres l'ingénieur Durand-Claye, le docteur Du Mesnil, et le statisticien Toussaint Loua, que ce projet est présenté par Bertillon : « Il existe à Paris un grand nombre de personnes qui vivent dans des logements beaucoup trop étroits. Sans même parler des malheureux qui vivent dans des garnis affreux où la même chambre sert à plusieurs dizaines de personnes, il y a beaucoup de familles d'ouvriers et d'ouvriers aisés où père, mère, garçons et filles vivent dans la même pièce. Il est aisé de deviner les conséquences d'un tel entassement au point de vue de l'hygiène et de la morale $»^{30}$. Ce principe de mesure statistique, motivé par la posture charitable héritée de la loi de 1850, définit le surpeuplement en terme de personnes par pièce et non selon la surface des logements. Après un long débat ${ }^{31}$ sur la nature des pièces à prendre en compte pour le calcul du peuplement la commission choisi finalement de recommander aux enquêteurs de ne désigner comme "pièce " que les locaux où il est possible d'installer un lit (soit une surface minimale de 2 mètres sur 1,50 mètres) et de demander aux ménages de signaler parmi toutes les « pièces » celles pourvues de cheminées.

Ces statistiques sur le surpeuplement vont non seulement permettre aux responsables parisiens de prendre conscience de l'état de l'habitat, notamment dans les anciennes communes suburbaines annexées en 1859, mais aussi de relever le niveau des besoins nécessaires dans le cadre de la construction de logements sociaux. Le critère de l'encombrement des ménages, souvent associé à celui de la dénatalité, va compter parmi les arguments développés en 1894, en faveur du vote de la loi Siegfried. À Paris, en 1912, lors du premier emprunt municipal en faveur du logement social, cet argument prévaut, non seulement pour fixer la répartition du nombre d'appartements à construire par arrondissement, mais aussi pour évaluer la dimension des futurs logements ${ }^{32}$.

\section{L'insalubrité réduite au milieu : la découverte des immeubles et îlots insalubres}

30 Mais si, dans le cadre du recensement, la mesure du surpeuplement s'attache comme la loi de 1850 le veut, à l'échelle de l'appartement et du ménage, tel n'est pas le cas des autres éléments de l'insalubrité -la présence de concessions d'eau ou de puits ainsi que de cabinets d'aisance particuliers et communs - que le recensement de 1891 mesure cette 
fois à l'échelle de l'immeuble. Ainsi, après de longs débats, les pouvoirs publics parisiens disposent d'une statistique sur le surpeuplement des ménages et le confort des immeubles ${ }^{33}$.

Cette double échelle de mesure du mauvais logement reflète la volonté des édiles qui attendent de leur service statistique l'identification précise des lieux et des milieux malsains que leur politique doit s'attacher à aménager. Dès 1883, le conseiller municipal Lamouroux propose à la commission permanente de statistique municipale de Paris "d'établir un relevé de toutes les conditions dans lesquelles se trouvent chacune des maisons de Paris au point de vue de la salubrité, de l'alimentation en eau, du système d'évacuation des vidanges, etc. ${ }^{34}$. Pour mettre en pratique la loi du 10 juillet 1894 relative à l'assainissement de Paris et de la Seine, obligeant les propriétaires à se raccorder au tout-à-l'égout, les élus de Paris souhaitent développer un système de carnets d'immeubles associant statistique médicale et gestion des réseaux urbains.

En 1894, Paul Juillerat (1854-1935), chef du service technique de l'assainissement et de l'habitation à la préfecture de la Seine, propose un véritable système d'informations (sur les 70000 immeubles parisiens) reposant sur le principe de relevés de terrain consignés dans des maisons classées dans des chemises de On trouve dans les chemises de maisons des relevés physiques relatifs au tissu urbain et sanitaire, notamment une enquête à l'intérieur de chaque bâtiment pour consigner la présence d'équipements, la propreté et l'état d'entretien des parties communes d'immeubles : cabinets d'aisance et ventilateurs, cours, escaliers, couloirs, écuries. Des données sur la nature de l'occupation identifient la présence d'établissements industriels ou commerciaux, le nombre de boutiques sur rue, le nombre d'habitants et surtout la statistique démographique et sanitaire provenant du service de la statistique municipale : nombre de décès par maladies transmissibles, dont la tuberculose et vers 1904 le cancer. À ce dossier sont joints, s'il y a lieu, les comptesrendus de visite des commissions des logements insalubres.

Les statistiques issues de l'exploitation du casier sanitaire permettent d'élaborer une théorie. Dans ces premiers essais Paul Juillerat montre que la mortalité par immeuble varie selon le degré d'équipement sanitaire ${ }^{35}$. Mais dès 1905 , il tend à relever que les plus fortes mortalités sont à imputer à l'absence d'air et de lumière, c'est-à-dire à la densité bâtie ${ }^{36}$. En effet, l'attention nouvelle pour la " phtisie pulmonaire », liée à l'émergence du mouvement pastorien, semble condamner les rues étroites et les cours obscures. Dès 1888, les médecins pastoriens Nocard et Roux déclarent pouvoir cultiver le bacille de Koch (1884) contenu dans des produits pathologiques comme le crachat et le pus. Si selon ces expériences, le bacille se développe beaucoup mieux à l'obscurité qu'à la lumière, dans le froid qu'à la chaleur humide, le casier sanitaire pourrait être le nouvel outil permettant d'exporter sur le terrain urbain ces découvertes de laboratoire. Ces principes permettent de détecter des immeubles insalubres, qualifiés de « maisons meurtrières » et regroupés dans des milieux particulièrement nocifs, les Ainsi, l'étude de l'influence de l'ensoleillement sur la maladie permet à Juillerat de constater dès 1904 qu'un nombre non négligeable de décès tuberculeux se concentrent depuis 1894 dans les mêmes maisons ${ }^{37}$. Le 8 mars 1906, le conseiller municipal Ambroise Rendu reprend mot pour mot une note technique de 1904 rédigée par Juillerat et précisant les caractéristiques, les délimitations, les classements en terme de risque de six îlots meurtriers, agglomérations d'immeubles, d'une dizaine à une trentaine de rues où le taux de mortalité par tuberculose, 800 pour 100000 habitants, selon les rapports du casier sanitaire, est le double de la moyenne 
parisienne. Dans ces îlots qui représentent 1600 maisons et 59000 logements, $80 \%$ des immeubles de ces secteurs sont considérés comme « tuberculeux »!

\section{Les facteurs sociaux de l'insalubrité}

34 L'expression "d'immeubles tuberculeux » est significative. Elle traduit l'importance accordée à la notion de milieu qui, dès lors, empêche de rechercher l'insalubrité dans le logement comme le voudrait la loi de 1850. Mais cette expression est aussi liée au concept de contagion qui porte l'attention sur les individus, porteurs de contages. Ces deux éléments résument toute l'ambiguité de la notion d'insalubrité à la fin $\mathrm{du}_{\mathrm{XIX}}{ }^{\mathrm{e}}$ siècle, réduite à des éléments techniques et des statistiques de mortalité, mais qui n'en continue pas moins à être un « marqueur social ».

D'après Juillerat: «presque jamais une maison à forte mortalité n'est isolée. Dans la presque totalité des cas, les maisons contiguës à des immeubles contaminés sont également frappées avec plus ou moins de sévérité. Le résultat est la formation de véritables îlots infectés ${ }^{38}$. Cette idée de contagion par le bâti a pour corollaire le refus d'une causalité sociale ${ }^{39}$ : «Pour nous la tuberculose n'est pas une maladie populaire. Ce n'est pas telle ou telle classe de la société qui est frappée de préférence à d'autres. Ce sont les habitants des logements sombres et mal aérés quelles que soient leurs conditions sociales, qui paient le plus lourd tribut $\aleph^{40}$. La quantification s'exerce en priorité sur l'immeuble tuberculeux susceptible d'en contaminer d'autres.

Néanmoins Juillerat ne manque pas de souligner combien la contagion urbaine peut provenir des individus vivant dans des îlots insalubres. Citant le professeur Louis Renon, auteur d'un ouvrage sur les maladies populaires et inquiet de l'augmentation de la densité des rapports sociaux en ville, Juillerat est conduit à se méfier de l'apparence anodine du tuberculeux éparpillant ses germes au gré de ses allées et venues ${ }^{41}$. La recherche des lieux de primo-infection, en l'occurrence les îlots insalubres, s'avère alors fondamentale ${ }^{42}$.

Enfin, on doit constater que les édiles identifient volontiers les îlots insalubres à l'implantation de populations indésirables. À proximité du marché des Halles, l'îlot SaintMerri $\left(3^{\mathrm{e}}\right.$ et $4^{\mathrm{e}}$ arrondissements) vient au premier rang des îlots mortels avec sa "population flottante» et ses garnis. Il est suivi par l'îlot Saint-Gervais (4 ${ }^{\mathrm{e}}$ arrondissement) dont les hôtels meublés et le " ghetto juif » à quelques pas de l'Hôtel de Ville renforcent la peur de la maladie. Pour Saint-Victor ( $5^{\mathrm{e}}$ arrondissement et îlot classé troisième), le nombre d'hôtels meublés inquiète autant que sa population marginale de clochards. En s'éloignant du centre, l'îlot Plaisance ( $14^{\mathrm{e}}$ arrondissement, îlot numéro 4) et Combat-Villette ( $19^{e}$, îlot numéro 5$)$ représentent l'habitat ouvrier des masures de la petite banlieue aux caractéristiques sociologiques proches de l'îlot Sainte-Marguerite $\left(11^{\mathrm{e}}\right.$ et $12^{\mathrm{e}}$, îlot classé sixième). Mais aucune statistique ne permet en dehors du recensement, peu exploité à cette échelle de l'îlot, d'établir les caractéristiques démographiques des habitants ${ }^{43}$.

\section{La réforme de la loi de 1850}

Ces éléments parisiens illustrent avec vigueur les caractéristiques de la loi de 1902 sur la protection de la santé publique dans les aspects qui concernent le logement et la réforme de la loi de 1850. L'institution d'un règlement sanitaire communal afin de prévenir les maladies transmissibles et d'assurer la salubrité des maisons, ainsi que celle d'un permis 
de construire dans les villes de plus de 20000 habitants sont introduites en 1902. Ces mesures montrent l'importance accordée à la notion d'immeuble dans le diagnostic d'insalubrité. Il s'agit non seulement de prescrire, mais aussi éventuellement de démolir, ce qui renvoie la théorie du « terrain » infectieux auquel s'attache le pastorisme.

Il n'en reste pas moins qu'une place non négligeable est accordée à la théorie contraire du « germe » non seulement par l'imposition de la vaccination - qui nous intéresse peu ici mais surtout par la modification de la définition de l'insalubrité établie depuis 1850 . En effet, on intègre désormais l'idée qu'est insalubre le lieu qui peut porter « atteinte à la vie et à la santé des occupants et des voisins ». Si l'on intègre à cette assertion la mise en place d'instruments de mesure comme les bureaux d'hygiène dans les villes de plus de 20000 habitants, tenus notamment de surveiller les taux de mortalité, nous ne pouvons que souligner combien la loi de 1902 confirme le rôle du milieu dans le développement de l'insalubrité, alors même que la loi de 1850 n'insistait finalement que sur les conditions de logement.

Mais si la loi de 1902 « inscrit de manière irréfutable le principe de la solidarité étroite qui unit les habitants en matière de salubrité et d'hygiène $»^{44}$ le maintien des solidarités ne peut s'exercer qu'à l'échelle du territoire communal. Ce modèle territorial explique pourquoi le législateur a voulu, comme en 1850, que "les applications de l'hygiène puissent varier selon les besoins hygiéniques locaux $»^{45}$. Ainsi, ce modèle territorial nous éloigne considérablement du modèle plutôt paternaliste de gestion de l'insalubrité qui ne s'intéressait qu'au ménage en danger de perdre la santé ou la vie, sans souci des voisins.

Comme l'a montré Florence Bourillon dans l'article précédent les débats préparatoires de la loi, révélaient des tendances contradictoires divisées entre un objectif de police sociale et une volonté d'assistance. Cette contradiction explique les effets limités de la loi. Dévorée par le localisme, elle se trouve confrontée à la difficulté de rationaliser les jugements d'insalubrité. Absorbée par la question du logement et de la famille, l'insalubrité se départit difficilement de la perspective médico-sociale pour intégrer la dimension technique, en particulier des réseaux sanitaires et du cadre bâti. Mais les prérogatives de puissance publique (décret de 1852 et loi de 1894) et les moyens d'expertise (casiers sanitaires) dont se sont dotés ingénieurs et agents-voyers permettent une évolution progressive de la loi de 1850 et l'apparition de nouveaux objets désignés comme insalubres (maisons meurtrières et îlots tuberculeux). Dans cette mutation la loi de 1850 permet l'émergence de deux nouveaux concepts : celui de surpeuplement qui permet d'aborder la question des normes et des besoins pour le logement social naissant à la fin du XIX ${ }^{\mathrm{e}}$ siècle (Loi Siegfried 1894), celui de la solidarité urbaine face à la contagion (loi de 1902) qui légitime l'expropriation pour cause d'insalubrité et la démolition des vieux quartiers populaires. Peu efficace, la loi de 1850 est tout de même au fondement des questions que pose encore l'urbanisme d'aujourd'hui.

\section{ANNEXES}

Loi du 13 avril 1850 relative à l'assainissement des logements insalubres ${ }^{46}$ 
Article premier - Dans toute commune où le conseil municipal l'aura déclaré nécessaire par une délibération spéciale, il nommera une commission chargée de rechercher et indiquer les mesures indispensables d'assainissement des logements et dépendances insalubres mis en location ou occupés par d'autres que le propriétaire, l'usufruitier ou l'usager.

Sont réputés insalubres les logements qui se trouvent dans des conditions de nature à porter atteinte à la vie ou à la santé de leurs habitants.

Article 2. - La commission se composera de neuf membres au plus, et de cinq au moins. En feront nécessairement partie un médecin et un architecte ou tout autre homme de l'art, ainsi qu'un membre du bureau de bienfaisance et du conseil des prud'hommes, si ces institutions existent dans la commune.

La présidence appartient au maire ou à l'adjoint.

Le médecin et l'architecte pourront être choisis hors de la commune.

La commune se renouvelle tous les deux ans par tiers ; les membres sortants sont indéfiniment rééligibles.

A Paris, la commission se compose de douze membres.

Article 3. - La commission visitera les lieux signalés comme insalubres. Elle déterminera l'état d'insalubrité, et en indiquera les causes, ainsi que les moyens d'y remédier. Elle désignera les logements qui ne seraient pas susceptibles d'assainissement.

Article 4. - Les rapports de la commission seront déposés au secrétariat de la mairie, et les parties intéressées mises en demeure d'en prendre communication et de produire leurs observations dans le délai d'un mois.

Article 5. - À l'expiration de ce délai, les rapports et observations seront soumis au conseil municipal, qui déterminera : 1) Les travaux d'assainissement et les lieux où ils devront être entièrement ou partiellement exécutés, ainsi que les délais de leur achèvement ; 2) Les habitations qui ne sont pas susceptibles d'assainissement.

Article 6. - Un recours est ouvert aux intéressés contre ces décisions devant le conseil de préfecture, dans le délai d'un mois à dater de la notification de l'arrêté municipal. Ce recours sera suspensif.

Article 7. - En vertu de la décision du conseil municipal ou de celle du conseil de préfecture, en cas de recours, s'il a été reconnu que les causes d'insalubrité sont dépendantes du fait du propriétaire ou de l'usufruitier, l'autorité municipale lui enjoindra, par mesure d'ordre et de police, d'exécuter les travaux nécessaires.

Article 8. - Les ouvertures pratiquées pour l'exécution des travaux d'assainissement seront exemptées, pendant trois ans, de la contribution des portes et fenêtres.

Article 9. - En cas d'inexécution, dans les délais déterminés, des travaux jugés nécessaires, et si le logement continue d'être occupé par un tiers, le propriétaire ou l'usufruitier sera passible d'une amende de seize francs à cent francs. Si les travaux n'ont pas été exécutés dans l'année qui aura suivi la condamnation, et si le logement insalubre a continué d'être occupé par un tiers, le propriétaire ou l'usufruitier sera passible d'une amende égale à la valeur des travaux, et pouvant être élevée au double.

Article 10. - S'il est reconnu que le logement n'est pas susceptible d'assainissement, et que les causes d'insalubrité sont dépendantes de l'habitation elle-même, l'autorité municipale pourra, dans le délai qu'elle fixera, en interdire provisoirement la location à titre d'habitation. 
L'interdiction absolue ne pourra être prononcée que par le conseil de préfecture, et, dans ce cas, il y aura recours de sa décision devant le Conseil d'État. Le propriétaire ou l'usufruitier qui aura contrevenu à l'interdiction prononcée sera condamné à une amende de seize à cent francs, et, en cas de récidive dans l'année, à une amende égale ou double de la valeur locative du logement interdit.

Article 11. - Lorsque, par suite de l'inexécution de la présente loi, il y aura lieu à la résiliation des baux, cette résiliation n'emportera en faveur du locataire aucuns dommages-intérêts.

Article 12. - L'article 463 du Code pénal sera applicable à toutes les contraventions cidessus indiquées.

Article 13. - Lorsque l'insalubrité est le résultat de causes extérieures et permanentes, ou lorsque ces causes ne peuvent être détruites que par des travaux d'ensemble, la commune pourra acquérir, suivant les formes et après l'accomplissement des formalités prescrites par la loi du 3 mai 1841, la totalité des propriétés comprises dans le périmètre des travaux.

Les portions de ces propriétés qui, après l'assainissement opéré, resteraient en dehors des alignements arrêtés pour les nouvelles constructions, pourront être revendues aux enchères publiques, sans que, dans ce cas, les anciens propriétaires ou leurs ayants droit puissent demander l'application des articles 60 et 61 de la loi du 3 mai 1841.

Article 14. - Les amendes prononcées en vertu de la présente loi seront attribuées en entier au bureau ou établissement de bienfaisance de la localité où sont situées les habitations à raison desquelles ces amendes auront été encourues.

\section{Décret relatif aux rues de Paris du 26 mars 1852 ${ }^{47}$.}

Article premier : Les rues de Paris continueront d'être soumises au régime de la grande voirie.

Article 2. - Dans tout projet d'expropriation pour l'élargissement, le redressement ou la formation des rues de Paris, l'administration aura la faculté de comprendre la totalité des immeubles atteints, lorsqu'elle jugera que les parties restantes ne sont pas d'une étendue ou d'une forme qui permette d'y élever des construire des constructions salubres. Elle pourra pareillement comprendre dans l'expropriation, des immeubles en dehors des alignements, lorsque leur acquisition sera nécessaire pour la suppression d'anciennes voies publiques jugées inutiles. Les parcelles de terrain acquises en dehors des alignements, et non susceptibles de recevoir des constructions salubres, seront réunies aux propriétés contiguës, soit à, l'amiable, soit par l'expropriation de ces propriétés, conformément à l'article 53 de la loi du 16 septembre 1807. La fixation du prix de ces terrains sera faite suivant les mêmes formes et devant la même juridiction que celle des expropriations ordinaires. L'article 58 de la loi du 3 mai 1841 est applicable à tous les actes et contrats relatifs aux terrains acquis pour la voie publique par simple mesure de voirie.

Article 3. - À l'avenir, l'étude de tout plan d'alignement de rue devra nécessairement comprendre le nivellement; celui-ci sera soumis à toutes les formalités qui régissent l'alignement. Tout constructeur de maison, avant de se mettre à l'œuvre, devra demander l'alignement et le nivellement de la voie publique au-devant de son terrain et s'y conformer. 
Article 4.- Il devra pareillement adresser à l'administration un plan et des coupes cotées des constructions qu'il projette, et se soumettre aux prescriptions qui lui seront faites, dans l'intérêt de la sûreté publique et de la salubrité. Vingt jours après le dépôt de ces plans et coupes au secrétariat de la préfecture de la Seine, le constructeur pourra commencer ses travaux d'après son plan, s'il ne lui a été notifié aucune injonction. Une coupe géologique des fouilles pour fondation de bâtiment sera dressée par tout architecte constructeur et remise à la préfecture de la Seine.

Article 5.- La façade des maisons sera constamment tenue en bon état de propreté. Elles seront grattées, repeintes ou badigeonnées au moins une fois tous les dix ans sur l'injonction qui sera faite au propriétaire par l'autorité municipale. Les contrevenants seront passibles d'une amende qui ne pourra excéder cent francs.

Article 6. - Toute construction nouvelle dans une rue pourvue d'égouts devra être disposée de manière à y conduire ses eaux pluviales et ménagères. La même disposition sera prise pour toute maison ancienne en cas de grosses réparations et en tous cas avant dix ans.

Article 7.-Il sera statué par un décret ultérieur, rendu dans la forme des règlements d'administration publique, en ce qui concerne la hauteur des maisons, les combles et les lucarnes.

Article 8.- Les propriétaires riverains des voies publiques empierrées supporteront les frais de premier établissement des travaux, d'après les règles qui existent à l'égard des propriétaires riverains des rues pavées.

Article 9. - Les dispositions du présent décret pourront être appliquées à toutes les villes qui en feront la demande, par des décrets spéciaux rendus dans la forme des règlements d'administration publique.

Article 10.- Le ministre de l'Intérieur, de l'Agriculture et du Commerce est chargé de l'exécution du présent décret qui sera inséré au Bulletin des lois.

\section{NOTES}

1. En tant que sociologue adoptant une démarche historienne, cette idée rejoint la perspective constructiviste développée par exemple par Christian TOPALOV, Naissance du chômeur, 1880-1900, Albin Michel, 1994, 624 p. Nous posons aussi pour notre part l'hypothèse que l'insalubrité est aussi un « objet construit » par les politiques, les rapports sociaux, les idéologies, l'évolution des catégories de pensée, etc... Le second intérêt sociologique de la loi de 1850 est qu'elle permet de soumettre à la question l'idéal-type de la bureaucratie développé par Max WEBER dans Économie et société, 1921, trad. fr. Paris, Plon, 1971, à savoir la compatibilité de la rationalisation avec la décentralisation des pouvoirs.

2. Voir aussi, pour une période plus large que la nôtre Lion MURARD et Patrick ZYLBERMAN, L'hygiène dans la République, la santé publique en France ou l'utopie contrariée, 1870-1918, Paris, Fayard, $805 \mathrm{p}$.

3. Pour un point de vue complémentaire voir Roger-Henri GUERRAND Propriétaires et locataires Les origines du logement social en France 1850-1914, Paris, Quintette, 1987, 345 p. ; Susanna MAGRI « Les propriétaires, les locataires, la loi. Jalons pour une analyse sociologique des rapports locatifs 1850-1920 ", Revue française de sociologie, no 3, 1998, pp. 397-417.

4. Présidées à Paris par le maire de l'arrondissement ces commissions se composent de médecins, pharmaciens, chimistes, vétérinaires parfois ingénieurs, industriels. Ils ont un rôle d'observation 
et de recommandations, ils visitent sur place suite à un dépôt de plainte les lieux désignés et tentent de concilier dans ce cadre les parties. Leur statut de bénévoles et leur appartenance à la notabilité, leur permet d'exercer un ascendant sur les locataires et les propriétaires. Nous verrons qu'à Paris leur rôle est à la fois complémentaire et concurrentiel de celui des commissions des logements insalubres.

5. En 1866, le préfet de la Seine introduit les séances d'une Commission des Logements Insalubres en relevant que la commission se trouve être un arbitre entre deux nécessités aussi fondamentales l'une que l'autre : la santé et la propriété.

6. Et celle-ci n'est possible que si le logement a été signalé. Néanmoins cette règle semble levée vers 1852-53 lors de l'épidémie de choléra, sous l'effet de la rumeur publique. Elsbeth KALF, L'hygienisation de la vie quotidienne. Le logement Insalubre à Paris, Thèse sous la direction de Nicole Haumont, Université Paris VII, 1995, fo 58 . Sur les processus à l'œuvre lors de l'épidémie de choléra : Patrice BOURDELAIS et Jean-Yves RAULOT, Une peur bleue : histoire du choléra en France 1832-1854, Paris, Payot, 1987, 310 p.

7. A. N., F8 211.

8. Néanmoins, il faut attendre le congrès international d'assainissement et de salubrité de l'habitation de Genève en 1906 pour que la question de la responsabilité du locataire soit soulevée.

9. Alain CORBIN, Le miasme et la jonquille. L'odorat et l'imaginaire social XVIII ${ }^{e}$ XIX ${ }^{e}$ siècles, Paris, Aubier-Montaigne, 1982, $335 \mathrm{p}$.

10. Paul JUILLERAT et André LEVY-DORVILLE, Note sur l'organisation et le fonctionnement du bureau de l'assainissement et de l'habitation, préfecture de la Seine, direction des affaires municipales, 1900, 65 p., p. 4 ; l'ouvrage reprend la jurisprudence de la commission des logements insalubres depuis 1851 ; voir aussi les Rapports de la commission des logements insalubres 1851-1870, A.D. Paris, VD 6-83 (par arrondissements) ; et les Rapports de la commission des logements insalubres de la Ville de Paris, A.N., F8 211.

11. Paul JUILLERAT et André LEVY-DORVILLE, Note sur l'organisation..., ouv. cité, p. 20.

12. Cette ordonnance est le fruit des recommandations du conseil d'hygiène. L'instruction qui accompagne cette ordonnance indique que l'insalubrité peut être soit locale (le logement de la famille) soit générale (provenant de la maison tout entière). Dans le premier cas, résoudre l'insalubrité conduit à s'intéresser à l'air, au mode de chauffage, à la propreté. Dans le second cas, la salubrité des maisons dépend de la présence de cabinets d'aisance communs, de l'évacuation des eaux ménagères. Lorsqu'ils instruisent une plainte les conseils d'hygiène doivent, si l'insalubrité leur semble fondée et le propriétaire récalcitrant à toute recommandation, transmettre le dossier à la commission des logements insalubres. On comprend que dans ce cadre, l'exigence des conseils d'hygiène ait des effets importants sur l'activité de la commission des logements insalubres et la nature des affaires qu'elle a à connaître.

13. Paul JUILLERAT et André LEVY-DORVILLE, Note sur l'organisation..., ouv. cité, p. 20.

14. Rapport de 1857, dans Paul JUILLERAT et André LEVY-DORVILLE, Note sur l'organisation..., ouv. cité, p. 17 ; en majuscule dans le texte ; voir aussi le rapport de 1866, p. 4, A.N., F8 211.

15. Selon le rapport du comité consultatif d'hygiène du 19 décembre 1870, A.N., F8 211, rapporteur Jean Isabelle, la loi de 1850 est en train de perdre son caractère moral et charitable.

16. En $1879,70 \%$ des affaires sont portées au Tribunal d'après Toussaint Loua.

17. Paul JUILLERAT et André LEVY-DORVILLE, Note sur l'organisation..., ouv. cité, p. 32.

18. Néanmoins c'est en 1880 que le Conseil de Préfecture annule deux prescriptions de la commission imposant à des propriétaires l'approvisionnement en eau de leurs immeubles.

19. Ministère de l'Agriculture et du Commerce, commission ministérielle dite des odeurs de Paris, Observations des ingénieurs du service municipal de Paris au sujet des projets de MM. Girard et Brouardel, Paris, Chaix, 1881. 
20. Cité par Octave DU MESNIL, «L'habitation du pauvre à Paris ", Revue d'hygiène, Paris, 1882, pp. 1-17.

21. Les fonctions de bénévoles ont tendance à disparaître tant pour les commissions d'hygiène (dans les années 1870) qui accordent des jetons de présence à leurs participants et les dédommagent lorsqu'ils rédigent un rapport que pour les commissions des logements insalubres vers la fin des années 1880 . Voir Elsbeth KALF, L'hygienisation..., ouv. cité. On assiste donc à une disparition progressive du caractère charitable de la loi au profit d'une vision plus technique, voire à un déplacement de la question sociale vers celle de l'assainissement.

22. Cité par Louis BERGERON et Marcel RONCAYOLO, Paris, genèse d'un paysage, Picard 1988, p. 236.

23. Toussaint LOUA, "Les logements insalubres de la ville de Paris », Journal de la société de statistique de Paris, tome 3, 1879, pp. 44-49.

24. Ou un renvoi au Conseil d'hygiène et de salubrité présidé par le préfet de police qui dispose éventuellement à son tour de la possibilité de saisir la commission des logements insalubres.

25. Voir Elsbeth KALF, L'hygienisation..., ouv. cité.

26. Annuaire statistique de la préfecture de la Seine, 1883.

27. Octave DU MESNIL, «Les garnis insalubres de Paris ", Annales d'hygiène publique et de médecine légale, tome 1, 1878. pp. 84-105.

28. Octave DU MESNIL, «L'habitation du pauvre à Paris ", Revue d'hygiène, 1882, p. 3.

29. Ibidem, p. 4.

30. Commission permanente de statistique municipale de Paris. Compte-rendu des débats 25 octobre 1881 , p. 5.

31. Ce débat qui soulève la question des critères d'habitabilité a été commenté par nous dans : «Deux statistiques pour décrire l'habitat populaire. Le recensement des conditions de logement et le casier sanitaire des maisons. Paris 1880-1906», le Mouvement social, janvier-mars 1998, pp. 79-96.

32. Yankel FIJALKOW, «Le surpeuplement ou la tuberculose. Deux critères pour identifier les mal-logés. Paris, 1850-1945. », dans Nicole HAUMONT et Jean-Pierre LEVY [dir.], la ville éclatée, quartiers et peuplements, Paris, L'Harmattan, 1998, pp. 113-127.

33. Les libellés du questionnaire de 1891 sont les suivants :

«FEUILLE DE MENAGE : Combien ce logement contient-il de pièces (sur la rue, sur la cour, sur rue et cour, sur le jardin.) ? Total des pièces. Combien sur ce total y a t-il de pièces à cheminée ?

BORDEREAU DE MAISON : Nombre de ménages. Nombre des personnes de la maison résidant habituellement dans la commune (Présentes/Absentes). Nombre de personnes de passage. Nombre des locaux d'habitation vacants. Nombre des locaux servant d'ateliers, de magasins ou de boutiques (les divers locaux occupés par un même locataire sont comptés pour un seul local). Nombre des étages au-dessus du rez-de-chaussée. Y a-t-il une concession d'eau dans la maison ? Y a-t-il un puits? Combien y a-t-il de cabinets d'aisance dans la maison? Particuliers? Communs?»

34. Commission permanente de statistique municipale, Comptes-rendus, 1883, p. 10.

35. Paul JUILLERAT, « Note statistique sur deux groupes de maisons de Paris d'après les données fournies par le casier sanitaire ", Revue d'hygiène et de police sanitaire, 20 janvier 1899.

36. Préfecture de la Seine, Rapports sur la répartition de la mortalité par tuberculose pulmonaire dans les maisons de Paris depuis le $1^{\text {er }}$ janvier 1894 jusqu'au $1^{\text {er }}$ janvier 1910, 1894-1908, 12 volumes. Le titre du volume change en 1905 : Rapports sur la répartition de la tuberculose pulmonaire dans les maisons de Paris; et en 1906 : Rapports sur la répartition de la mortalité par tuberculose pulmonaire et par cancer dans les maisons de Paris.

37. Rapports sur la répartition de la tuberculose pulmonaire dans les maisons de Paris, 1905, p. 59.

38. Idem.

39. «Les quartiers où la tuberculose s'établit à demeure sont les quartiers où les logis sont obscurs et mal aérés. Toutes les autres causes invoquées jusqu'ici, la misère, l'alcoolisme, le vice 
sous toutes ses formes sont des causes secondaires si on en compare l'effet à la privation d'air et surtout de la lumière solaire", Paul JUILLERAT, Rapports sur la répartition de la tuberculose pulmonaire dans les maisons de Paris, 1906.

40. Paul JUILLERAT «L'habitation urbaine ", Premier congrès international d'assainissement et de salubrité de l'habitation, Paris, 1905, p. 45.

41. Cité par Paul JUILLERAT dans : Une institution nécessaire : le casier sanitaire des maisons de Paris, 1906, p. 12.

42. Alain Cottereau souligne aussi ce parti pris écologique alors que l'usure du travail aurait pu être l'une des causes étudiées à l'époque ; Alain COTTEREAU, « la tuberculose à Paris, 1882-1914 : maladie urbaine ou maladie du travail ? Un exemple de production de connaissances et de méconnaissances sur les modes de vie ", dans Vie quotidienne en milieu urbain, supplément aux Annales de la recherche urbaine, Paris 1978 ; voir aussi Lion MURARD et Patrick ZYLBERMAN, « Les murs qui tuent ", Les cahiers médico-sociaux, Genève, no 4, 1983, pp. 285-294.

43. Il faut attendre 1937 pour que le conseiller municipal Louis Sellier présente un rapport très complet comprenant quelques éléments statistiques (très rudimentaires) provenant du recensement de 1936. Rapport sur la question des logements insalubres, Conseil municipal de Paris, 1937.

44. Paul STRAUSS et Alfred FILLASSIER, Loi sur la protection de la santé publique. Travaux législatifs, guide pratique et commentaire, Jules Roussel, 1905. p. 60.

45. Rapport au Sénat de Victor Cornil, 27 décembre 1895.

46. J.-B. DUVERGIER, Collection complète des lois, décrets, ordonnances, règlements et avis du conseil d'État, tome 50, année 1850, pp. 131-133.

47. M. ALPHAND [dir.], Recueil des lois, ordonnances, décrets et règlements relatifs aux alignements, à l'expropriation pour cause d'utilité publique spécialement dans les voies de Paris, Paris, Imprimerie Nouvelle, 1886, pp. 154-156.

\section{RÉSUMÉS}

La spécificité de la loi de 1850 consiste dans l'imprécision de la définition de l'insalubrité et dans les pouvoirs nouveaux accordés aux municipalités. Ce texte, qui mobilise dans un premier temps les notables locaux, fait l'objet d'applications très différentes selon les communes. Le mouvement de professionnalisation des métiers concernés par le traitement de l'insalubrité conduit à une normalisation de la définition de l'insalubrité. Un nouvel objet de préoccupation sociale apparaît : le logement, le ménage, l'habitat. À Paris, l'insalubrité des logements est étudiée avec attention, mais dès 1894 une perspective inverse se développe plus centrée sur les milieux et îlots insalubres. Celle-ci annonce la réforme de la loi de 1850 par la loi relative à la santé publique de 1902.

The notion of unhealthiness towards rationalisation 1850-1902. The specificity of the 1850 law consistes in the indistinctness of the definition of insalubrity and the new powers granted to Municipalities. This text, which initially mobilized local autorities, was the object of very different enforcements according to the municipalities. The movement of professionalisation of the trades concerned with the treatment of insalubrity led to a standardisation of the definition of insalubrity. A new object of social preoccupation appeared: dwellings, housekeeping. In Paris, insalubrity of flats was studied with attention, but from 1894 one inverse perspective developed 
more centred on unhealthy places. This one announced the reform of the 1850 law by the 1902 public health act.

INDEX

Mots-clés : Paris, Second empire, Troisième République, Histoire urbaine, Logement, Histoire culturelle, Histoire sociale

\section{AUTEUR}

\section{YANKEL FIJALKOW}

Maître de conférences à l'Université Paris VII et chercheur au Centre de recherche sur l'habitat/ LOUEST, UMR 7544 du CNRS.

cy.fijalkow[at]wanadoo.fr 